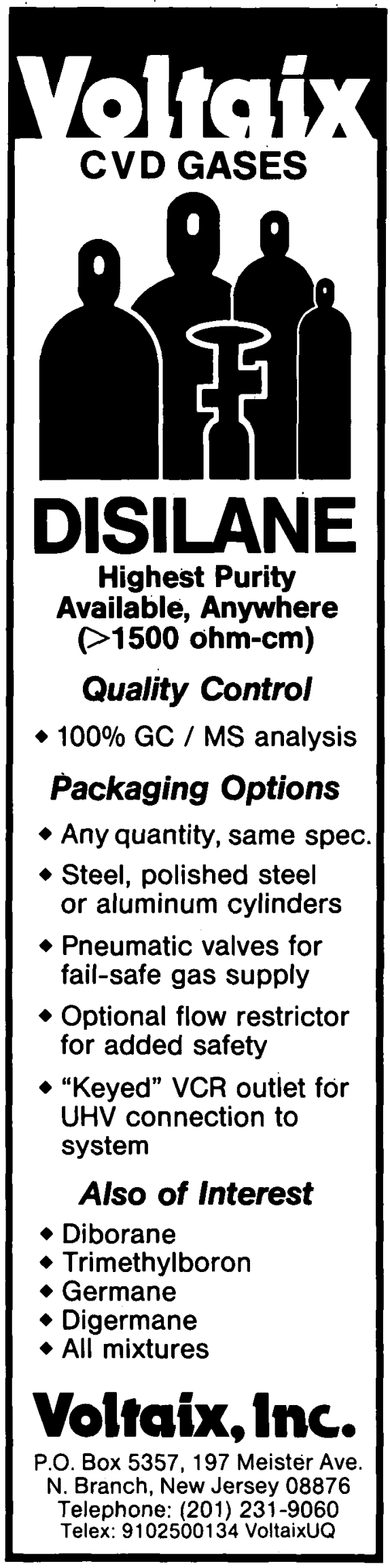

Please visit Booth No. 614 at the MRS Show in San Francisco, April 17-19, 1990.

\title{
Fall '89 Meeting
}

Features Broad Mix of Topics More Highlights from Boston

The February 1990 issue of the MRS Bulletin reported on the highlights of some of the events and presentations featured at the 1989 Fall Meeting in Boston. (See "Boston Meeting Attracts Worldwide Audience" p. 56-60 in the February MRS Bulletin.) This issue completes the report on the 1989 Fall Meeting with some final symposium highlights.

For more details readers are encouraged to order the MRS Symposium Proceedings or Extended Abstracts volumes from the MRS Publications Department, 9800 McKnight Road, Pittsburgh, PA 15237; telephone (412) 367-3012; fax (412) 367-4373. In the few instances where proceedings will not be published, readers should contact one of the symposium organizers for further details.

\section{Tailored Interfaces in Composite Materials (Symposium N)}

MRS Symposium Proceedings, Vol. 170

Organizers: Carlo G. Pantano (Pennsylvania State University), Eric H.J. Chen (E.I. duPont de Nemours \& Co., Inc.)

Support: Air Force Office of Scientific Research; E.I. duPont de Nemours $\mathcal{E}$ Co., Inc. .

This symposium brought together over 80 international researchers from government laboratory, universities, and industry, with diverse backgrounds ranging from physics and chemistry to engineering mechanics, and with interests in a wide variety of materials including polymers, metals and ceramics. Presentations centered on tailoring interfaces in order to optimize the adhesion or transfer of load between reinforcing phases and the matrix, enhance crack deflection through debonding, or control interface reactivity. The symposium was designed to be interdisciplinary, and some of the sessions also addressed the micromechanics, chemistry, and characterization of interfaces in general.
Optical Fiber Materials and Processing (Symposium P) MRS Symposium Proceedings, Vol. 172 Organizers: James W. Fleming (AT\&T Bell Laboratories), George H. Sigel Jr. (Rutgers University), Shiro Takahashi (NTT Corporation), Paul W. France (British Telecom Research Laboratory).

Support: Air Products; Alcatel NV; ATET Bell Laboratories; Coming Glass Works; DeSoto, Inc.; Heraeus Amersil; Johnson \& Johnson; Nippon Mining Co. Ltd.; Nippon Telegmaph \& Telephone Corporation; Schott Fiber Optics, Inc.; Tosoh USA, Inc.

This 1989 symposium expanded the interest and attendance of the first MRS symposium on this topic at the 1986 MRS Fall Meeting, and provided an excellent gathering of experts who are exploring optical fiber for the 1990s and beyond. The aim of this symposium was to assemble investigators with both optical fiber materials and processing interests to share their expertise, whether their goals addressed production of silica-based fiber for low-cost applications, long wavelength materials for ultralow loss or power transmission, broad transmission wavelength fiber for spectroscopy, nonlinear optics, or other related objectives.

Fifty-two papers were presented. Topics included those addressed in the 1986 symposium, dealing with processing various materials for several types of optical fibers. Additionally, since the 1986 meeting, there have been increased interest and significant scientific advancements in defects in high-silica glasses, nonlinear optical effects (particularly for fiber amplifiers), and hermetic coatings, and these topics were addressed in the 1989 symposium. Optical fiber has been developed to such a degree that it can now be seen as the transmission medium of choice for a broad range of applications from telecommunications through spectroscopy. The continued suc- 
cess of lightwave transmission systems and the development of the new applications will depend on continued materials and processing research in a number of areas.

\section{Materials Synthesis Utilizing}

Biological Processes (Symposium R) MRS Symposium Proceedings, Vol. 174 Organizers: P.C. Rieke (Battelle-Pacific Northwest Laboratory), P.D. Calvert (University of Arizona), M. Alper (University of Califormia, Berkeley).

Support: Air Force Office of Scientific Research; Office of Basic Energy Sciences, U.S. Department of Energy.

Talks in this first MRS symposium devoted entirely to the study of materials made using biological processes covered the properties of materials produced by living organisms, the methods by which those materials are produced, and the techniques by which those methods could be controlled, modified or reproduced in artificial systems. More than 50 presentations covered four major areas of research: biomineralization, synthesis of polymeric materials by organisms, enzymatic synthesis of materials in cell-free systems and selfassembly in natural and synthetic systems.

Biomineralization is of interest in a strict materials context because it represents an alternative way of making high performance composites. S. Mann (Bath, England), L. Addadi and S. Weiner (Weizmann, Israel), and members of the Nancollas group (SUNY, Buffalo) discussed the ways organisms exert control over the mineralization process. The implicit challenge is to find a way to translate this control to synthetic systems. Studies aimed at putting this translation into practice were also described. In principle we might be able to harness simple organisms such as bacteria or yeast to manufacture ceramic particles in fermenters. R. Blakemore discussed the formation of magnetite in Aquaspirillum, which use the Earth's magnetic field to distinguish the vertical direction in the anaerobic environment of lake beds.

Another theme of this symposium was to explore the possibility of mimicking biological materials in synthetic structures. A particular early goal for this work is the development of tougher, more damageresistant composite structures. If we are to attempt to reproduce biological structures in synthetic materials we need to convince ourselves that these structures are indeed strong. This is not a simple question since the strength must be related to some base material value. We do not expect any calcium carbonate composite to be as strong

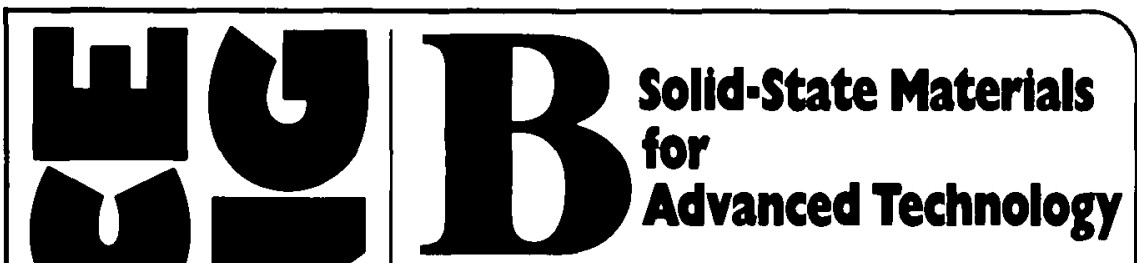

A timely new journal on electronic materials research and semiconductor physics

\section{Editors}

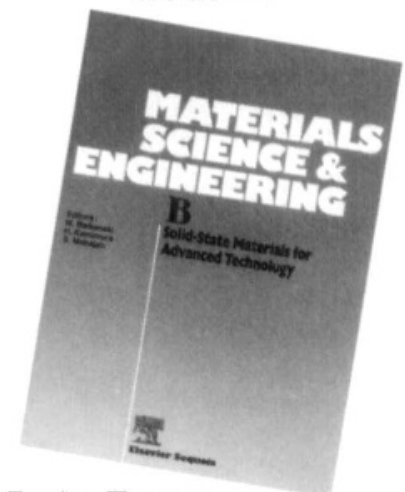

M. Balkanski, Paris, France

H. Kamimura, Tokyo, Japan

S. Mahajan, Pittsburgh, PA, USA

This international Journal covers the full range of topics pertaining to the expanding field of solid-state physics and materials science of electronic materials.

The Journal covers processing, characterization and structure of materials both in bulk and in thin films.

Materials include:

* Semiconductors

* Superconductors

* Low-Dimensional Compounds

* Magnetic and Optoelectronic Materials

* Fast Ion Conductors

\section{ELSEVIER SEQUOIA SA}

P.O. Box 564

1001 Lausanne 1

Switzerland

Tel. : 021/207381

For customers in USA and Canada:

Elsevier Science Publishing Co., Inc.

Attn.: Journal Information Center

655 Avenue of the Americas

NEW YORK, NY 10010, USA

Tel.: (212) 633-3750

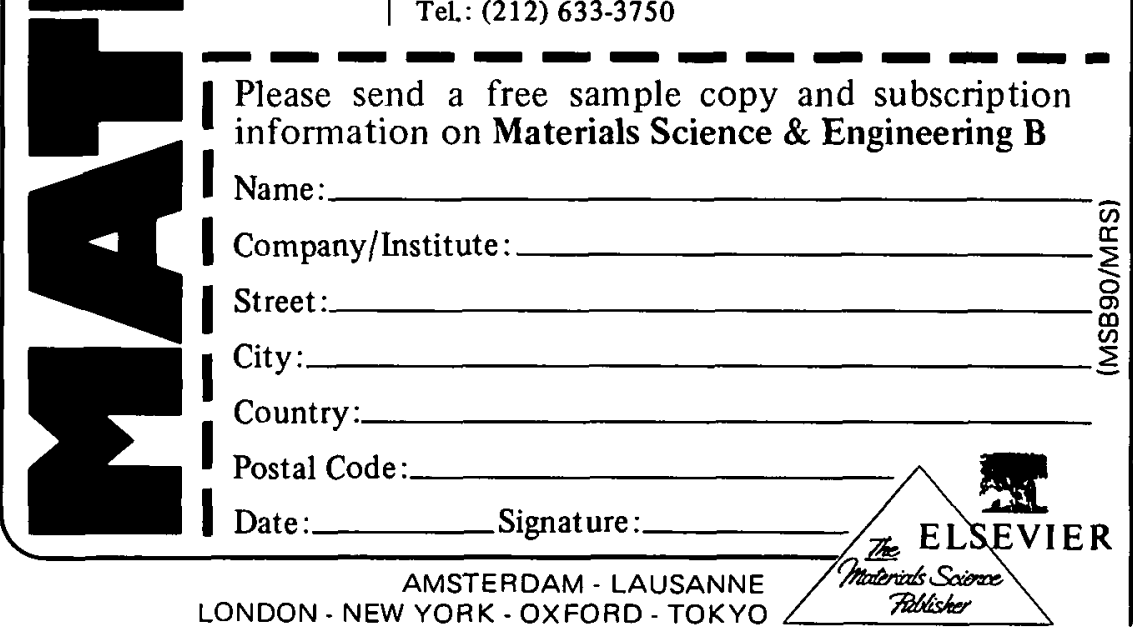

Please visit Booth Nos. 418 \& 420 at the MAS Show in San Francisco, April 17.19, 1990. 
as titanium. M. Sarakiya and I. Aksay (University of Washington) and V.J. Laraia and A.H. Heuer (Cast Western) are looking at the microstructure and fracture properties of shell in comparison with synthetic composites.

G. Whitesides (Harvard) opened the session on enzymatic synthesis, outlining the impressive breadth of molecular structures, properties and applications of the materials synthesized by living organisms. Several speakers discussed the production of organic polymers produced by living organisms, highlighting some that already have commercial application. The copolymers of polyhydroxybutyrate (PHB) are in commercial production by bacterial fermentation. Many water-soluble polymers are biologically produced, but PHB represents the first attempt to make a plastic by fermentation. If the material can be made reproducibly and competitive with conventional plastics it would open the door for biotechnology in materials. As Whitesides stressed, one advantage of biological polymers is that very complex chain structures can be made, with controlled copolymerization and branching. Harnessing biological polymerization mechanisms could move us into a new regime of "designer" polymers.

The use of purified enzymes to create or modify materials has expanded greatly in the past few years and is no longer restricted to chiral resolution or the synthesis of small molecules for pharmaceutical or chemical use. M. Bednarski (Lawrence Berkeley Lab) described the enzymatic and chemical modification of silicon surfaces in the control of surface properties and the production of sensors. Other researchers described novel polyesters with controlled structures that could be achieved using lipases.

An afternoon was devoted to "fooling with Mother Nature." J. Bibbs (Lawrence Berkeley Lab/Scripps) described his work with $\mathrm{C}-\mathrm{H}$. Wong on the chemical modification of proteolytic enzymes to alter their reaction specificity. The work of J. Kirsch (Lawrence Berkeley Lab) was presented, demonstrating the controlled engineering of the amino acid sequences of enzymes to alter both their substrate specificity and also their stability at higher temperatures. D. Suich (Lawrence Berkeley Lab) reported on work he performed with $P$. Schultz on a novel technique that allows insertion of non-natural amino acids into enzymes, greatly broadening the range of modifications that could be achieved. $D$. Urry (U. Alabama, Birmingham) described the elastic properties of polypeptides he had synthesized. On the gene-making front, several groups described the synthe- sis of genes to direct the production of new or modified materials, including silks, collagen, adhesives and those with optoelectronic properties. A talk on the final day (J. Sivak, University of Waterloo) discussed the structure of the eye lens.

Several presentations on biopolymers and synthetic polymers focused on the mechanisms for achieving self-assembly. A fascinating video by $N$. Mendelson and J. Thwaites showed how inhibiting cellwall cleavage during cell division leads to formation of living fibers which repeatedly grow in length, twist into a helix, and fold over to repeat the process on a larger scale. Nature's ability to create complex structure through self-assembly will be a process of keen interest to materials scientists in the future. H.S. Yoon (KAIST, Korea) discussed an apparent case of self-assembly in polyaramid fibers.

The whole philosophy of materials processing and design is different in the living organisms. Some day the natural and synthetic approaches will merge, but at the moment we can learn a lot by exploring the differences. This symposium will also be a part of the 1990 MRS Fall Meeting. The organizers expect the 1990 program to include an even broader representation of the field. They also hope to attract more traditional "materials scientists" and begin to establish biological processes as one set of processing techniques among many in the production of useful materials.

\section{Fractal Aspects of Materials (Symposium T)}

Extended Abstract Volume EA-20

Organizers: J.H. Kaufman (IBM T.J. Watson Research Center), James E. Martin (Sandia National Laboratories), P.W. Schmidt (University of Missouri), B.B. Mandelbrot (IBM T.J. Watson Research Center).

Support: Army Research Office; Office of $\mathrm{Na}$ val Research; Ford Motor Company; Exxon Research and Engineering Company; Cabot Corporation; E.I. duPont de Nemours \& Company, Inc.; IBM Corponation; SchlumbergerDoll Research.

The sixth annual MRS symposium on the application of fractal concepts to materials was marked by the continued development of new ideas in many diverse areas. Although this diversity added enormously to the interest of the meeting, it impedes a facile summary of the technical highlights; thus the following is a rather fractal description of the symposium.

As usual, pattern formation continued to be a topic of interest, with excellent papers on the formation of droplets on surfaces, and the self-organized criticality that results from the avalanche of these drop- lets when a critical droplet size is attained (the so-called "Beer Stein" problem). A paper on spinodal decomposition demonstrated that in a system with long-range interactions nucleation occurs on a fractal cluster, rather than on the classical Euclidean droplet.

Papers on fractally rough surfaces were of keen interest this year, with many talks on the formation of such surfaces through fracture and deposition processes. Scanning tunneling microscopy gave investigators the opportunity to amaze the audience with visually striking images of rough surface topography (no broken probes were reported), while others reported reciprocal space, small angle $x$-ray scattering studies of fractal surfaces. Papers in the joint session with the symposium on the Scientific Basis for Nuclear Waste Management (Symposium V) discussed the flow through fractal fracture networks in an effort to understand transport of nuclear waste through geologic media.

The electrochemistry contingent regaled the audience with the complex morphologies that can result from electrodeposition under various applied voltages. And on the theoretical side, a solution to the problem of the complex double layer impedance of a fractally rough electrode was presented, providing a theoretical basis for the constant phase angle impedances electrochemists have historically observed.

Finally, a Friday morning session on the dynamics of gels attracted a large, albeit enervated, audience who came to hear about the strange fractal dynamics exhibited at the sol-gel transition. This dynamics is readily manifested in the relaxation of either externally imposed shear stresses or thermally driven density fluctuations, and can only be described by a self-similar cascade of relaxation processes.

In all, the symposium was well attended and the discussions energetic, while the occasional vituperative outburst added an edge of anticipation to the usually staid presentations. It is apparent that the application of fractal concepts to materials problems is still a rapidly growing field that attracts expertise from a broad spectrum of the materials community.

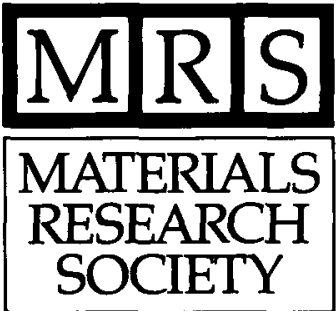

